\title{
Inhibition of Herpes Simplex Viruses, Types 1 and 2, by Ginsenoside 20(S)-Rg3 5
}

\author{
Stephen M. Wright ${ }^{*}$ and Elliot Altman
}

Department of Biology and the Tennessee Center for Botanical Medicine Research, Middle Tennessee State University, TN 37132, USA

\author{
Received: August 26, 2019 \\ Revised: November 2, 2019 \\ Accepted: November 2, 2019 \\ First published online: \\ November 6, 2019 \\ ${ }^{*}$ Corresponding author \\ Phone: +615-898-2056 \\ Fax: +615-898-5093 \\ E-mail: Stephen.Wright@mtsu.edu \\ S upplementary data for this \\ paper are available on-line only at \\ http://jmb.or.kr. \\ pISSN 1017-7825, eISSN 1738-8872 \\ Copyright(C) 2020 by \\ The Korean Society for Microbiology \\ and Biotechnology
}

\begin{abstract}
Infections by herpes simplex viruses have an immense impact on humans, ranging from selflimiting, benign illness to serious, life-threatening diseases. While nucleoside analog drugs are available, resistance has been increasing and currently no vaccine exists. Ginsenosides derived from Panax ginseng have been documented to inhibit several viruses and bolster immune defenses. This study evaluated 12 of the most relevant ginsenosides from $P$. ginseng for toxicities and inhibition of herpes simplex viruses types 1 and 2 in Vero cells. The effects of test compounds and virus infection were determined using a PrestoBlue cell viability assay. Time course studies were also conducted to better understand at what points the virus life cycle was affected. Non-toxic concentrations of the ginsenosides were determined and ranged from $12.5 \mu \mathrm{M}$ to greater than $100 \mu \mathrm{M}$. Ginsenoside 20(S)-Rg3 demonstrated the greatest inhibitory effect and was active against both HSV-1 and HSV-2 with an $\mathrm{IC}_{50}$ of approximately $35 \mu \mathrm{M}$. The most dramatic inhibition-over 100\% compared to controls-occurred when the virus was exposed to 20(S)-Rg3 for $4 \mathrm{~h}$ prior to being added to cells. 20(S)-Rg3 holds promise as a potential chemotherapeutic agent against herpes simplex viruses and, when used together with valacyclovir, may prevent increased resistance to drugs.
\end{abstract}

Keywords: Ginsenoside Rg3, Panax ginseng, herpes simplex viruses, valacyclovir, virus inhibition

\section{Introduction}

The Herpesviridae family is comprised of three subfamilies designated alpha, beta and gamma. Herpes simplex viruses types 1 and 2 (HSV-1 and HSV-2) are included within the alpha herpesviruses. HSV-1 is most commonly associated with oral infection and is reportedly present in nearly $70 \%$ of humans [1]. Other syndromes frequently associated with HSV-1 infection include keratitis and herpes gladiatorum, or skin disease in wrestlers. HSV-2 is more frequently associated with genital infection and may result in neonatal disease. In addition to these mucocutaneous infections, more severe, life-threatening disease may occur. In the United States, herpes simplex viruses are responsible for nearly $20 \%$ of encephalitis cases [2]. As reviewed [3], there is evidence for an association between HSV-1 and Alzheimer's disease. Herpes simplex viruses are able to cause latent infections in neurons with periodic reactivations throughout life. No vaccine exists to prevent infection by the herpes simplex viruses [4].

The first step in infection of a host is attachment of viral glycoprotein to a host receptor. Five different herpes simplex glycoproteins may be involved in attachment to host cells [5]. Entry into the host cell is initiated by binding with heparan sulfate and seems to involve a complex process of sequential binding of viral proteins with other host proteins [6]. Once the viral DNA is in the host nucleus, genome expression occurs in differential order. Alpha genes enhance expression of beta genes for replication followed by structural gamma genes [7]. Assembled capsids typically bud through the nuclear membrane.

Acyclovir, acting as a nucleoside analog, has been a useful chemotherapeutic intervention for HSV-1 and 2 infections since it is activated by viral thymidine kinase [8]. However, resistance to acyclovir has been increasing and is particularly problematic among immunocompromised 
individuals [9]. It would be advantageous to find additional therapeutic agents that could curtail herpes infection. Natural products have long been recognized as potential sources for antiviral compounds [10]. Panax ginseng Meyer has served as a traditional Chinese medicine for centuries [11]. Extracts of ginseng have been described as having numerous benefits, including anti-inflammatory effects [12], prevention of obesity [13], and anti-cancer activity [14]. Red ginseng has been reported to inhibit a wide variety of viruses including respiratory (influenza, rhinoviruses), gastrointestinal (enteroviruses, rotaviruses, noroviruses), and hepatic (both hepatitis A and B viruses) [15]. As reviewed, [16], ginsenoside $\operatorname{Rg} 3$ reduced the severity of HSV-induced keratitis and decreased herpes plaque formation in cultured human amniotic cells.

Over 100 saponins or ginsenosides have been isolated from ginseng, which are generally divided into two groups, protopanaxadiols and protopanaxatriols [17]. Well known protopanaxadiols include $\mathrm{Rb} 1, \mathrm{Rb} 2, \mathrm{Rb} 3, \mathrm{Rc}, \mathrm{Rd}$, Rg3, Rh1, Rh2 and Rs1 while well known protopanaxatriols include Re, Rf, Rg1, Rg2 and Rh1. Some ginsenosides exist as $\mathrm{R}$ or $\mathrm{S}$ isoforms $20(\mathrm{R})$ or $20(\mathrm{~S})$ depending on the position of the hydroxyl moiety at carbon 20 [18]. Of the ginsenosides evaluated in this study, Rg2, Rg3, Rh1 and Rh2 occur as isoforms.

In this study, the most prevalent ginsenosides found in natural (white) and steamed (red) ginseng [19, 20] were evaluated for their antiviral activity against HSV-1 and HSV-2. These included Rb1, Rb2, Rb3, Rc, Rd, Re, Rf, Rg1, 20(R)-Rg2，20(S)-Rg2，20(R)-Rg3，20(S)-Rg3，20(R)-Rh1， 20(S)-Rh1, 20(R)-Rh2 and 20(S)-Rh2. The 20(R)-Rg2, 20(S)Rg2, 20(R)-Rg3, 20(S)-Rg3, 20(R)-Rh1, 20(S)-Rh1, 20(R)-Rh2 and $20(\mathrm{~S})-\mathrm{Rh} 2$ ginsenosides are conversion products that are generated when white ginseng is converted by steaming into red ginseng. We report that 20(S)-Rg3 demonstrated strong inhibitory activity against both herpes simplex viruses. Our results suggest that 20(S)-Rg3 affects some aspect associated with the interaction of the virus and host receptor proteins.

\section{Materials and Methods}

\section{Cells}

Vero cells (American Type Culture Collection (ATCC), USA, CCL-81) were maintained in medium 199 (Sigma Chemical Company, USA) that was supplemented with $8 \%$ fetal bovine serum (Invitrogen, USA), $1.25 \mu \mathrm{g} / \mathrm{ml}$ fungizone (Invitrogen) and penicillin-streptomycin, $1,000 \mathrm{U} / \mathrm{ml}$ and $1 \mathrm{mg} / \mathrm{ml}$ respectively (Sigma). Cells were passed weekly using $0.1 \%$ trypsin (Sigma) to detach the cells from the flasks. Cell quantification was done by trypan blue exclusion.

\section{Test Chemicals}

Twelve of the most prevalent ginsenosides, including the $\mathrm{R}$ and $\mathrm{S}$ isoforms for the four ginsenosides that had $\mathrm{R}$ and $\mathrm{S}$ confirmations, were evaluated for anti-herpes simplex activity. These included Rb1，Rb2，Rb3，Rc, Rd, Re, Rf, Rg1, 20(R)-Rg2, 20(S)-Rg2, 20(R)Rg3，20(S)-Rg3，20(R)-Rh1，20(S)-Rh1，20(R)-Rh2 and 20(S)-Rh2. Additionally the ginsenoside metabolites 20(R)-protopanaxadiol and 20(S)-protopanaxadiol were also tested, because of the similarity in structure to $20(\mathrm{R})-\mathrm{Rg} 3$ and $20(\mathrm{~S})-\mathrm{Rg} 3$, differing only by the lack of the disaccharide sophorose. These chemical structures are shown in Fig. 1. Structures of all ginsenosides evaluated in this study are provided as Supplemental Information, in Fig. S1. Valacyclovir (Sigma), a known anti-herpes compound, was used as a control for herpes inhibition. All compounds derived from P. ginseng were obtained from Biopurify Phytochemicals Ltd. at $98 \%$ purity (China) and were prepared in DMSO.

\section{Viruses and Quantification}

Herpes simplex virus type 1 (HSV-1, MacIntyre strain, ATCC, VR-539) or herpes simplex virus type 2 (HSV-2, ATCC, VR-734) were used in these studies. Virus titer was determined by plaque assay. Briefly, following $2 \mathrm{~h}$ adsorption of various virus dilutions onto a Vero cell monolayer in a 24 -well plate, the medium was removed and cells were overlaid with $1.5 \%$ carboxymethylcellulose (Sigma) and incubated $48 \mathrm{~h}$ at $37^{\circ} \mathrm{C}$ with $5 \% \mathrm{CO}_{2}$. After incubation, cells were fixed with methanol, stained with Giemsa (Sigma) and plaques were counted. Each virus dilution was prepared in quadruplicate.

The plaque assay procedure also was used to evaluate the percent plaque reduction; the only procedural change was that a

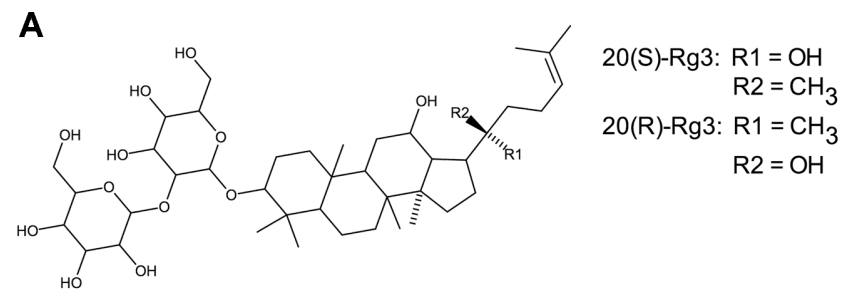

B

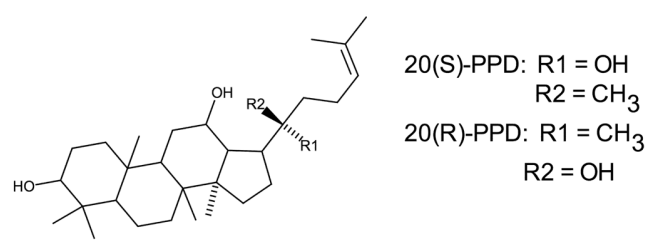

Fig. 1. Structures of ginsenoside $\mathrm{Rg} 3$ and protopanaxadiol. (A) 20(S)- and 20(R)-Rg3 chemical structures are shown. (B) 20(S)and 20(R)-protopanaxadiol (PPD) chemical structures are shown. 
constant virus concentration was used (50 PFU/well). Plaque reduction was measured by comparing the control (virus only), with valacyclovir $(16 \mu \mathrm{M})$ with virus or $20(\mathrm{~S})-\operatorname{Rg} 3(50 \mu \mathrm{M})$ with virus. Sample wells were evaluated in quadruplicate. Following the $2 \mathrm{~h}$ virus adsorption, the methylcellulose-medium containing the same concentrations of valacyclovir or 20(S)-Rg3 was added to the appropriate wells. After $48 \mathrm{~h}$, plaques were fixed, stained and counted.

\section{Cytotoxicity and Virus Inhibition}

All ginsenosides, isomers, and protopanaxadiols were evaluated for toxic effects on Vero cells prior to anti-viral testing. Cells were seeded at 5,000 cells/well in a black 96-well plate with clear bottom in a volume of $100 \mu \mathrm{l}$ and allowed to attach for $24 \mathrm{~h}$. The $P$. ginseng compounds were prepared in DMSO at $100 \mu \mathrm{M}$ and were taken through serial two-fold dilutions to determine the concentration when the rate of cell death was less than $10 \%$ compared to control, untreated cells. Each dilution was tested in three wells and all assays were repeated three times. Following $48 \mathrm{~h}$ incubation at $37^{\circ} \mathrm{C}$ in $5 \% \mathrm{CO}_{2}, 11 \mu \mathrm{l}$ of PrestoBlue (Invitrogen) was added to each well and the plate was incubated for an additional $30 \mathrm{~min}$. PrestoBlue determines cell viability by changing from blue to red as resazurin is reduced to resorufin by viable cells. Following the PrestoBlue incubation, fluorescence was determined by spectrophotometric reading using Soft Max Pro (Molecular Diagnostics, USA).

For cytotoxicity testing, $4 \mu \mathrm{l}$ of dilutions of the ginsenosides or protopanaxadiols were added to $396 \mu \mathrm{l}$ of medium 199 and $100 \mu \mathrm{l}$ of this mixture was added to each of three wells. For anti-viral testing, $4 \mu \mathrm{l}$ of the non-toxic reagent and $4 \mu \mathrm{l}$ of HSV-1 or HSV-2 diluted to an MOI of 0.1 were added to $392 \mu \mathrm{l}$ of medium 199 and $100 \mu \mathrm{l}$ of this was added to each of three wells. Virus inhibition was determined by comparing the cell death of reagent-exposed cells with cell death of control cells only receiving virus. The 50\% inhibitory concentration $\left(\mathrm{IC}_{50}\right)$ was determined through a series of two-fold dilutions and calculation by linear regression analysis.

\section{Time Studies}

For virus inhibition studies described above, reagent 20(S)-Rg3 and virus were added to cells simultaneously. In an attempt to evaluate the potential mode of action of 20(S)-Rg3, various combinations of valacyclovir, 20(S)-Rg3 and HSV-1 were tested at different time periods. The following combinations were evaluated at both one hour and four hours:

-Virus applied to cells first (for one hour or four hours), followed by addition of valacyclovir, 20(S)-Rg3 or both valacyclovir and 20(S)-Rg3 to the cells

- Valacyclovir or 20(S)-Rg3 or both valacyclovir and 20(S)-Rg3 applied to cells first (for one or four hours), followed by virus

-Valacyclovir or 20(S)-Rg3 incubated together with virus first for one or four hours then added to cells

All procedures described for evaluation of virus inhibition were followed in identical fashion as outlined for the PrestoBlue procedure except valacyclovir was used at a constant concentration of $16 \mu \mathrm{M}$ and $20(\mathrm{~S})-\mathrm{Rg} 3$ at $50 \mu \mathrm{M}$. The use of valacyclovir at $16 \mu \mathrm{M}$, or $5.76 \mu \mathrm{g} / \mathrm{ml}$, is in the reported $\mathrm{IC}_{50}$ ranges for this drug, from $0.02-13.5 \mu \mathrm{g} / \mathrm{ml}$ (New Drug Application \#20-487/S-007, GlaxoSmithKline, available from https://www.accessdata.fda.gov/ drugsatfda_docs/label/2005/020487S007rel2_lbl.pdf).

\section{Statistical Analysis}

All values are shown as the mean \pm standard error. A one-tailed Student's t-test was used to determine significance between groups. The level for significance was $\mathrm{p}<0.05$.

\section{Results}

\section{Toxicity}

All ginsenosides and the closely-related metabolites 20(R) and 20(S)-protopanaxadiol were tested for toxic effects on Vero cells. Both protopanaxadiol isoforms were the most

Table 1. Cytotoxicity and inhibition of HSV-1 by ginsenosides and protopanaxadiols.

\begin{tabular}{lcc}
\hline $\begin{array}{c}\text { Ginsenosides \& } \\
\text { Protopanaxadiols }\end{array}$ & $\begin{array}{c}\text { Concentration for } \\
\text { Cytotoxicity Testing } \\
(\mu \mathrm{M})\end{array}$ & $\begin{array}{c}\text { Percent Inhibition } \\
\text { of HSV-1 }\end{array}$ \\
\hline $\mathrm{Rb1}$ & 50 & 23.3 \\
$\mathrm{Rb} 2$ & 100 & 26.4 \\
$\mathrm{Rb3}$ & 100 & 30.9 \\
$\mathrm{Rc}$ & 100 & 28.6 \\
$\mathrm{Rd}$ & 100 & 36.1 \\
$\mathrm{Re}$ & 100 & 26.7 \\
$\mathrm{Rf}$ & 100 & $(38.0)^{\mathrm{a}}$ \\
$\mathrm{Rg} 1$ & 100 & 13.2 \\
20(S)-Rg2 & 100 & 44.3 \\
20(R)-Rg2 & 100 & 25.3 \\
20(S)-Rg3 & 100 & 90.3 \\
20(R)-Rg3 & 100 & 22.7 \\
20(S)-Rh1 & 100 & 14.2 \\
20(R)-Rh1 & 100 & 26.3 \\
20(S)-Rh2 & 25 & 46.2 \\
20(R)-Rh2 & 50 & 12.0 \\
20(S)-protopanaxadiol & 12.5 & $(1.7)$ \\
20(R)-protopanaxadiol & 12.5 & 14.4 \\
\hline
\end{tabular}

The non-toxic concentration was defined as causing less than $10 \%$ cell death compared with controls and was used to determine virus inhibition. To determine cytotoxicity, all compounds were initially tested starting at $100 \mu \mathrm{M}$. Subsequent 2-fold dilutions were made as necessary.

${ }^{a} \mathrm{~A}$ value in parentheses indicates that the non-toxic concentration with virus resulted in a higher percentage of cell death than the control that was exposed to the virus alone. 
Table 2. Cytotoxicity of 20(S)-Rg3 against Vero cells.

\begin{tabular}{cc}
\hline Concentration $(\mu \mathrm{M})$ & Percent Cell Viability \\
\hline 50 & $(113.0 \pm 3.1)^{\mathrm{a}}$ \\
100 & $(114.3 \pm 4.5)$ \\
125 & $(122.3 \pm 1.8)$ \\
250 & $(128.7 \pm 1.4)$ \\
500 & $17.7 \pm 14.7$ \\
1,000 & $0.4 \pm 0.02$ \\
\hline
\end{tabular}

${ }^{a}$ Values in parenthesis indicate greater cell proliferation or metabolic activity compared to untreated control cells.

toxic compounds and had to be diluted to $12.5 \mu \mathrm{M}$ to cause less than 10 percent cell death compared to controls (Table 1). The majority of compounds tested resulted in less than $10 \%$ cell death when used at the highest starting concentration of $100 \mu \mathrm{M}$. Since 20(S)-Rg3 was determined to effectively inhibit herpes simplex viruses, additional testing was done to find the actual concentration that was toxic to Vero cells, starting at 1,000 $\mu \mathrm{M}$ and making 2-fold dilutions (Table 2). When used at 1,000 $\mu \mathrm{M}, 20(\mathrm{~S})-\operatorname{Rg} 3$ resulted in less than $1 \%$ viable cells. Less than $20 \%$ of cells remained viable when $\operatorname{Rg} 3$ was used at $500 \mu \mathrm{M}$. Subsequent two-fold dilutions were non-toxic and at concentrations of $250 \mu \mathrm{M}$ and less, exposure to $20(\mathrm{~S})-\mathrm{Rg} 3 \mathrm{had}$ a proliferative effect on cells or at least enhanced metabolic activity compared to untreated cells. The non-toxic concentration of all compounds shown in Table 1 was used for virus inhibition tests. The known herpes simplex inhibitor valacyclovir was used at $16 \mu \mathrm{M}$; cells retained nearly $99 \%$ viability at this concentration $(1.2 \pm 0.4$ percent cell death occurred).

\section{Virus Inhibition}

For all ginsenosides, isoforms, or metabolites, only 20(S)Rg3 was able to dramatically inhibit HSV-1 (Table 1). While 20(S)-Rh2 inhibited HSV-1 by $46.2 \%$, it was approximately half as effective as 20(S)-Rg3. Given the effectiveness of 20(S)-Rg3, additional investigations were carried out with this molecule. When coupled with virus, one of the

Table 3. Inhibition of herpes simplex viruses by 20(S)-Rg3.

\begin{tabular}{ccc}
\hline $\begin{array}{c}\text { 20(S)-Rg3 } \\
\text { Concentration } \mu \mathrm{M}\end{array}$ & $\begin{array}{c}\text { Percent Inhibition } \\
\text { of HSV-1 }\end{array}$ & $\begin{array}{c}\text { Percent Inhibition } \\
\text { of HSV-2 }\end{array}$ \\
\hline 25 & $11.0 \pm 2.6$ & $14.3 \pm 3.3$ \\
50 & $82.3 \pm 3.5$ & $81.7 \pm 10.3$ \\
100 & $90.3 \pm 9.9$ & $114.7 \pm 6.7$ \\
\hline
\end{tabular}

The values are the percent inhibition \pm the standard error compared to the control cells that were exposed to the viruses but not to 20(S)-Rg3.

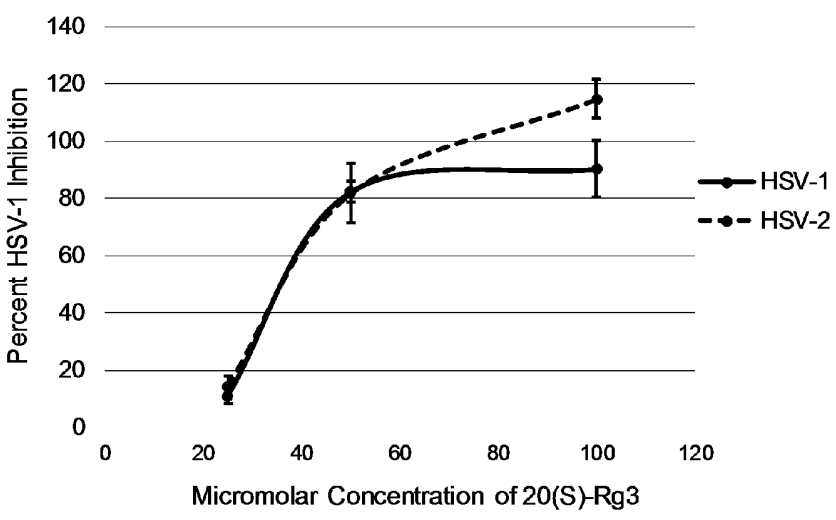

Fig. 2. Dose response curve for 20(S)-Rg3.

For both HSV-1 and HSV-2, the $\mathrm{IC}_{50}$ was approximately $35 \mu \mathrm{M}$.

ginsenosides, Rf, and the metabolite 20(S)-protopanaxadiol resulted in greater cell death than virus alone, even when used at a non-toxic level.

Due to the effective antiviral activity of 20(S)-Rg3 against HSV-1, 20(S)-Rg3 was also evaluated for inhibition of HSV2. As evident in Table 3, both of these herpes alphaviruses were nearly completely inhibited when 20 (S)-Rg3 was used at $100 \mu \mathrm{M}$. At $50 \mu \mathrm{M}$, each virus was suppressed by $80 \%$. The $\mathrm{IC}_{50}$ of 20(S)-Rg3 was determined to be approximately $35 \mu \mathrm{M}$ as shown in Fig. 2. As a control, valacyclovir had an $\mathrm{IC}_{50}$ concentration of $13.2 \mu \mathrm{M}$ against HSV-1.

Plaque reduction of HSV-1 by 20(S)-Rg3 was also tested to evaluate bioactivity. Table 4 shows the percent reduction of HSV-1 by valacyclovir (used at $16 \mu \mathrm{M}$ ) and 20(S)-Rg3 $(50 \mu \mathrm{M})$. All wells were infected with the same concentration of virus (approximately 50 PFU each) but only $1.5 \pm 0.3$ plaques were visible on cells treated with $20(\mathrm{~S})-\mathrm{Rg} 3$.

\section{Time Studies}

The length of time for exposure of cells to virus or to bioactive compound was tested. As shown in Fig. 3, when cells were exposed to HSV-1 before treatment with either valacyclovir $(16 \mu \mathrm{M})$ or $20(\mathrm{~S})-\operatorname{Rg} 3(50 \mu \mathrm{M})$, virus inhibition was reduced. When virus was present on cells $4 \mathrm{~h}$ prior to 20(S)-Rg3 addition, the percent inhibition was only $50.7 \pm$

Table 4. Plaque reduction of HSV-1.

\begin{tabular}{lcc}
\hline & Number of Plaques & Percent Reduction \\
\hline Control & $56.7 \pm 1.2$ & \\
Valacyclovir & $8.1 \pm 1.1$ & 85.7 \\
$20(\mathrm{~S})-\mathrm{Rg} 3$ & $1.5 \pm 0.3$ & 97.3 \\
\hline
\end{tabular}

The number of plaques \pm the standard error and the percent reduction compared to the control after treatment by valacyclovir or 20(S)-Rg3 are shown. 


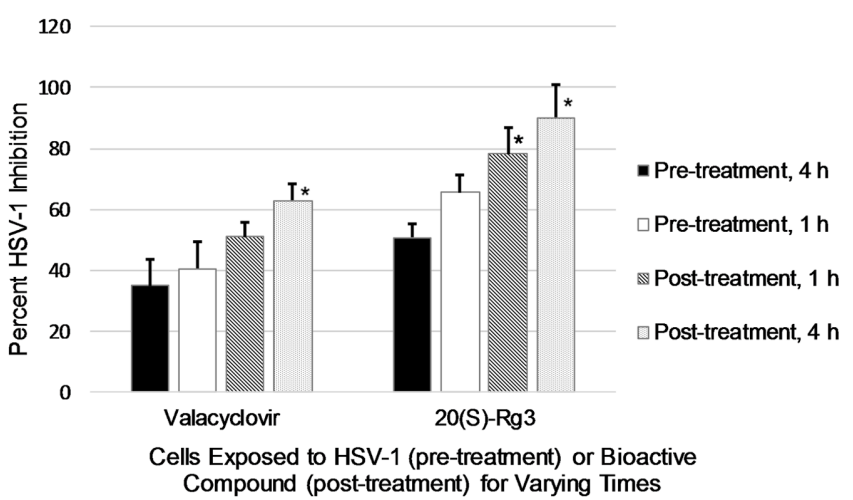

Fig. 3. Percent HSV-1 inhibition.

Cells were exposed to virus before the addition of bioactive compounds (pre-treatment; $4 \mathrm{~h}=$ black bars, $1 \mathrm{~h}=$ white bars) or exposed to bioactive compounds prior to virus being added (posttreatment; $4 \mathrm{~h}=$ stippled bars, $1 \mathrm{~h}=$ hatched bars). The asterisks indicate significant differences between $4 \mathrm{~h}$ pre-treatment values and post-treatment, $p<0.05$.

4.4. When cells were treated with $20(\mathrm{~S})-\mathrm{Rg} 3$ prior to HSV-1 addition, the virus was inhibited more effectively. As the length of time for bioactive reagent pretreatment increased to $4 \mathrm{~h}$, virus inhibition also increased, reaching a maximum percent inhibition of $90.1 \pm 11.1$ for 20(S)-Rg3. Posttreatment with $20(\mathrm{~S})-\mathrm{Rg} 3$ at both 1 and $4 \mathrm{~h}$ resulted in significantly increased virus inhibition $(p<0.05)$ compared to pre-treatment with virus for $4 \mathrm{~h}$.

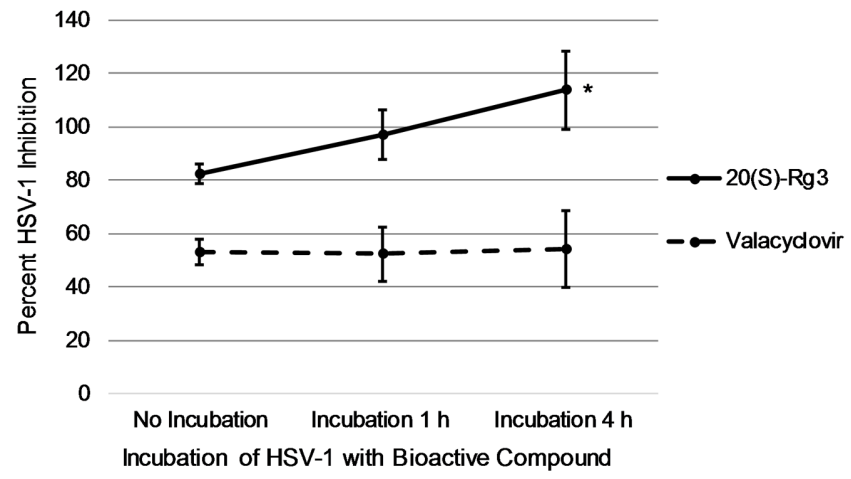

Fig. 4. Percent inhibition of HSV-1 following incubation of virus with bioactive compounds.

HSV-1 and the bioactive compound, valacyclovir or (20)S-Rg3, were applied directly to cells simultaneously (no incubation) or the virus and compound were incubated together for $1 \mathrm{~h}$ or $4 \mathrm{~h}$ prior to being added to the cells. A significant difference exists between the incubation of HSV-1 and 20(S)-Rg3 for $4 \mathrm{~h}$ compared with no incubation, $p<0.05$. No differences existed for incubation of HSV-1 and valacyclovir.
When HSV-1 was incubated together with valacyclovir prior to addition to cells, there was no change in virus inhibition, ranging from 52.3 to 54.7 percent from 0 to $4 \mathrm{~h}$ (Fig. 4). However, as incubation of HSV-1 with 20(S)-Rg3 increased to $4 \mathrm{~h}$, there was a dramatic increase in inhibition of virus to $113.7 \pm 14.8$ percent $(p<0.05)$. When virus was incubated together $4 \mathrm{~h}$ with $20(\mathrm{~S})-\operatorname{Rg} 3$ and valacyclovir simultaneously, a synergistic effect was evident with percent virus inhibition at $125.3 \pm 4.9$.

\section{Discussion}

In this study the 12 most prevalent ginsenosides from natural (white) or steamed (red) ginseng were examined as potential inhibitors of HSV-1 and HSV-2. 20(S)-Rg3 demonstrated significant antiviral activity with an $\mathrm{IC}_{50}$ of approximately $35 \mu \mathrm{M}$ against both HSV-1 and HSV-2. Additionally, 20(S)-Rg3 had very low toxicity, with an $\mathrm{IC}_{50}$ value of approximately $400 \mu \mathrm{M}$ against the host Vero cells. With a selectivity of 11 -fold, 20(S)-Rg-3 appears to be very promising as an anti-viral agent against HSV-1 and HSV-2.

The ginsenoside metabolite 20(S)-protopanaxadiol has been reported to have pharmacological activity [21] and is structurally similar to ginsenoside 20(S)-Rg3. Thus it seemed prudent to include protopanaxadiol in toxicity and anti-herpes investigations. The concentration of 20(S)protopanaxadiol found to be non-toxic in this study was $12.5 \mu \mathrm{M}$ which is in agreement with Bak [22] who determined that a concentration of $20 \mu \mathrm{M}$ was not toxic for PC12 cells. Protopanaxadiol has been found to be an effective anti-oxidant that preserves healthy mitochondrial function [22] as well as providing protection for UVassociated skin wrinkling [21]. However, in this study, both protopanaxadiol isoforms failed to have any protective effect against HSV-1.

Several ginsensoides, both protopanaxadiols and protopanaxatriols, have been reported to have anti-viral effects. Rb1 decreased the apoptotic effects of HSV-1 in a human glioma cell line [23]. In that study, to achieve inhibition of herpes a concentration of $400 \mu \mathrm{g} / \mathrm{ml}$ was necessary. We found that Rb1 caused greater than 10\% Vero cell death when used at a concentration higher than $50 \mu \mathrm{M}$ and that minimal inhibition (23\%) of HSV-1 occurred at this non-toxic concentration. Chen et al. reported [24] that Rh2 suppressed tumor growth in hepatitis B virus hepatocellular carcinoma cells. The protopanaxatriols Re, Rf, and Rg2 had inhibitory effects on coxsackie B3 virus and human rhinovirus 3 at $100 \mu \mathrm{g} / \mathrm{ml}$ [25]. Ginsenoside-Re has been reported to have anti- 
influenza virus activity due to enhancing immune responses after infection in mice [26]. None of these ginsenosides exhibited at least $50 \%$ inhibition of HSV-1 in our investigation.

One of the bioactive properties attributed to $\mathrm{Rg} 3$ includes anti-cancer effects by decreasing drug efflux from a cancer cell line [27]. In that study, Rg3 was cytotoxic for human carcinoma cells KB V20C when used at $120 \mu \mathrm{M}$ but this concentration had no effect on normal WI 38 cells. Rg3 has been determined to have potent anti-inflammatory activity by down-regulation of cytokine expression [28]. Rg3 has also been recognized to inhibit several virus life cycles. 20(S)-Rg3, but not 20(R)-Rg3, was reported to prevent rotavirus infection in mice, presumably due to mediation of immune responses [29]. Studies have shown that $\operatorname{Rg} 3$ reduces the expression of epidermal growth factor receptor on cells [30]. Such down-regulation of host receptors may impact viral attachment to a potential host. Kim [14] described $\operatorname{Rg} 3$ as a potential therapeutic agent for hepatitis $\mathrm{C}$ virus. Rg3 restored normal mitochondrial processes that hepatitis $C$ had disrupted, thus helping prevent chronic virus infection. $\operatorname{Rg} 3$ also has been reported to inhibit hepatitis B virus capsid maturation by interfering with proinflammatory cytokine expression [31]. Kang et al. [32] determined that $\mathrm{Rg} 3$ prevented the replication of a murine gammaherpes virus, likely through inhibition of cellular signaling pathways. A crude extract of Korean red ginseng has been documented to inhibit HSV-1 vaginal infection in mice [33]. The mechanism for the protective effects of the ginseng extract was suggested to involve stimulation of immune responses, particularly interferon $\gamma$ and natural killer cells. In the current study, purified 20(S)-Rg3 was shown to effectively curtail replication by both HSV-1 and HSV-2. Decreased host cell death and plaque reduction by $20(S)-\operatorname{Rg} 3$ indicate reduced success for completion of the herpes life cycle.

What may 20(S)-Rg3 be doing to virus-host interaction? The experiments shown in Fig. 3 demonstrated the therapeutic effects of $20(\mathrm{~S})-\operatorname{Rg} 3$, as well as valacyclovir, were strongest when cells were treated with the bioactive compounds before virus had an opportunity to attach and penetrate its host. This is in line with the known mechanism of valacyclovir as a nucleoside analog. Valacyclovir enters cells but remains inactive until HSV is also present. Increased anti-viral activity is seen with preexposure of cells to valacyclovir; upon viral infection, valacyclovir is already present, "waiting" to be activated. In relation to 20(S)-Rg3, while all aspects of herpes simplex attachment and penetration of host cells have not been fully elucidated, a good picture of the process has been described. Host receptor heparan sulfate is bound by herpes glycoproteins $\mathrm{gB}$ and $\mathrm{gC}$ [34]. The next step involves binding of $\mathrm{gD}$ and the glycoprotein dimer $\mathrm{gH}-\mathrm{gL}$ with the Herpes Virus Entry Mediator receptors and nectin-1 or nectin-2, which are cell adhesion molecules [6]. In suggesting the effects of $20(S)-\operatorname{Rg} 3$, the data from Fig. 3 allow a possible contributing mechanism for this ginsenoside. It has been reported that exposure of endothelial cells to $\mathrm{Rg} 3$ resulted in decreased expression of adhesion molecules, both vascular cell adhesion molecule-1 and intracellular cell adhesion molecule-1 [28]. Optimal receptors for HSV attachment include the cell adhesion molecules nectin-1 and nectin-2. Further, Uchida and colleagues [35] investigated HSV-1 attachment and penetration using mutations in $\mathrm{gB}$ and $\mathrm{gD}$. When a mutation in $\mathrm{gD}$ prevented normal binding with the Herpes Virus Entry Mediator, the virus was still able to penetrate the host cell through an epidermal growth factor receptor. Thus, a possible mechanism of 20(S)-Rg3 inhibition of herpes simplex viruses may relate to binding with such alternate receptors since $\mathrm{Rg} 3$ has been reported to downregulate epidermal growth factor receptor [30].

A second possible mechanism of $20(S)-\operatorname{Rg} 3$ is suggested by the experiments shown in Fig. 4, where the virus was incubated with the bioactive compounds prior to exposure to host cells. For valacyclovir, there was no difference in anti-viral activity whether or not virus was exposed to valacyclovir prior to infecting cells. This is expected since valacyclovir is only activated by viral thymidine kinase once the virus is replicating inside the host cell. However, when $20(S)-\operatorname{Rg} 3$ was allowed to interact for $4 \mathrm{~h}$ with the virus prior to exposure to host cells, successful production of virus was reduced significantly over $110 \%$. Given the array of at least five possible attachment glycoproteins, it is reasonable to hypothesize that incubation of HSV-1 with 20(S)-Rg3 prior to exposure to host cells may result in ginsenoside binding with a viral attachment protein, decreasing the opportunity for viral penetration. While the possible impact of 20(S)-Rg3 on viral entrance to host cells is speculative, there is little doubt that $20(\mathrm{~S})-\operatorname{Rg} 3$ has a pronounced effect on the successful completion of the viral life cycle, particularly evident by the dramatic reduction in PFUs (Table 4).

Another promising aspect for chemotherapeutic use of 20(S)-Rg3 is suggested by the synergistic effect that occurred when the ginsenoside was used together with valacyclovir, inhibiting virus activity by $125 \%$. Using two pharmacological agents with differing modes of action 
would not only be effective at inhibiting herpes simplex virus, it would also be useful in combating increasing resistance to acyclovir.

\section{Acknowledgments}

We thank Destaalem T. Kidane and Brock A. Arivett for assistance with preparation of Figures. We also acknowledge GreenWay Herbal Products, LLC, for providing funding for this research.

\section{Conflict of Interest}

The authors have no financial conflicts of interest to declare.

\section{References}

1. Faral-Tello P, Mirazo S, Dutra C, Perez A, Geis-Asteggiante L, Frabasile S, et al. 2012. Cytotoxic, virucidal, and antiviral activity of South American plant and algae extracts. Sci. World J. 2012: 174837.

2. Bookstaver PB, Mohorn PL, Shah A, Tesh LD, Quidley AM, Kothari R, et al. 2017. Management of viral central nervous system infections: a primer for clinicians. J. Cent. Nerv. Syst. Dis. 9: 1-12.

3. Hogestyn JM, Mock DJ, Mayer-Proschel M. 2018. Contributions of neurotropic human herpesviruses herpes simplex 1 and human herpesvirus 6 to neurodegenerative disease pathology. Neural. Regen. Res. 13: 211-221.

4. Koelle DM. 2006. Vaccines for herpes simplex virus infections. Curr. Opin. Investig. Drugs 7: 136-141.

5. Akhtar J, Shukla D. 2009. Viral entry mechanisms: cellular and viral mediators of herpes simplex virus entry. FEBS J. 276: 7228-7236.

6. Sedy JR, Spear PG, Ware CF. 2008. Cross-regulation between herpesviruses and the TNF superfamily members. Nat. Rev. Immunol. 8: 861-873.

7. Sandri-Goldin RM, Goldin AL, Holland LE, Glorioso JC, Levine M. 1983. Expression of herpes simplex virus beta and gamma genes integrated in mammalian cells and their induction by an alpha gene product. Mol. Cell Biol. 3: 20282044.

8. Kuo YC, Lin LC, Tsai WJ, Chou CJ, Kung SH, Ho YH. 2002. Samarangenin B from Limonium sinense suppresses herpes simplex virus type 1 replication in Vero cells by regulation of viral macromolecular synthesis. Antimicrob. Agents Chemother. 46: 2854-2864.

9. Piret J, Boivin G. 2011. Resistance of herpes simplex viruses to nucleoside analogues: mechanisms, prevalence, and management. Antimicrob. Agents Chemother. 55: 459-472.
10. Chattopadhya D, Chawla-Sarkar M, Chatterjee T, Dey RS, Bag P, Chakraborti S, et al. 2009. Recent advancements for the evaluation of anti-viral activities of natural products. New Biotechnol. 25: 347-368.

11. Coleman CI, Hebert JH, Reddy P. 2003. The effects of Panax ginseng on quality of life. J. Clin. Pharm. Ther. 28: 5-15.

12. Dragos D, Gilca M, Gaman L, Vlad A, Iosif L, Stoian I, et al. 2017. Phytomedicine in joint disorders. Nutrients 9: 1-18.

13. Chen G, Li H, Zhao Y, Cai E, Gao Y, Liu S, et al. 2017. Saponins from stems and leaves of Panex ginseng prevent obesity via regulating thermogenesis, lipogenesis and lipolysis in high-fat diet-induced obese C57BL/6 mice. Food Chem. Toxicol. 106: 393-403.

14. Kim SJ, Jang JY, Kim EJ, Cho EK, Ahn DG, Kim C, et al. 2017. Ginsenoside Rg3 restores hepatitis C virus-induced aberrant mitochondrian dynamics and inhibits virus propagation. Hepatology 66: 758-771.

15. Im K, Kim J, Min H. 2016. Ginseng, the natural effectual antiviral: protective effects of Korean red ginseng against viral infection. J. Ginseng Res. 40: 309-314.

16. Xin-Mei C, Chang-Zheng Z, Xiao-Ping Z. New progress on the pharmacological and pharmacokinetical study of ginsenoside Rg3. J. Drug Metabol. Toxicol. 3: 114.

17. Shin B-Y, Kwon SW, Park JH. 2015. Chemical diversity of ginseng saponins from Panax ginseng. J. Ginseng Res. 39: 287298.

18. Peng M, Yi YX, Zhang T, Ding Y, Le J. 2018. Stereoisomers of saponins in Panax notoginseng (Sanqi): a review. Front. Pharmacol. 9: 188.

19. Sun S, Qi L-W, Du G-J, Mehendale SR, Wang C-Z, Yuan CS. 2011. Red notoginsneng: higher ginsenoside content and stronger anticancer potential than Asian and American ginseng. Food Chem. 125: 1299-1305.

20. Ha J, Shim Y-S, Seo D, Kim K, Ito M, Nakagawa H. 2013. Determination of 22 ginsenosides in ginseng products using ultra-high-performance liquid chromatography. J. Chromatogr. Sci. 51: 355-360.

21. Han S, Lim TG, Kim JE, Yang H, Oh DK, Yoon Park JH, et al. 2017. The ginsenoside derivative 20(S)-protopanaxadiol inhibits solar ultraviolet light-induced matrix metalloproteinase1 expression. J. Cell Biochem. 118: 3756-3764.

22. Bak DH, Kim HD, Kim YO, Park CG, Han SY, Kim JJ. 2016. Neuroprotective effects of 20(S)-protopanaxadiol against glutamate-induced mitochondrial dysfunction in PC12 cells. Int. J. Mol. Med. 37: 378-386.

23. Liang YY, Wang B, Qian DM, Li L, Wang ZH, Hu M, et al. 2012. Inhibitory effects of ginsenoside $\mathrm{Rb} 1$ on apoptosis caused by HSV-1 in human glioma cells. Virol. Sin. 27: 1925.

24. Chen W, Qiu Y. 2015. Ginsenoside Rh2 targets EGFR by upregulation of miR-491 to enhance anti-tumor activity in hepatitis B virus-related hepatocellular carcinoma. Cell. Biochem. Biophys. 72: 325-331. 
25. Song JH, Choi HJ, Song HH, Hong EH, Lee BR, Oh SR, et al. 2014. Antiviral activity of ginsenosides against coxsackievirus B3, enterovirus 71, and human rhinovirus 3. J. Ginseng Res. 38: 173-179.

26. Song X, Chen J, Sakwiwatkul K, Li R, Hu S. 2010. Enhancement of immune responses to influenza vaccine (H3N2) by ginsenoside Re. Int. Immunopharmacol. 10: 351356.

27. Kwon HY, Kim EH, Kim SW, Kim SN, Park JD, Rhee DK. 2008. Selective toxicity of ginsenoside $\operatorname{Rg} 3$ on multidrug resistant cells by membrane fluidity modulation. Arch. Pharm. Res. 31: 171-177.

28. Hien TT, Kim ND, Kim HS, Kang KW. 2010. Ginsenoside Rg3 inhibits tumor necrosis factor-alpha-induced expression of cell adhesion molecules in human endothelial cells. Pharmazie 65: 699-701.

29. Yang H, Oh KH, Kim HJ, Cho YH, Yoo YC. 2018. Ginsenoside- $\mathrm{Rb} 2$ and 20(S)-ginsenoside-Rg3 from Korean red ginseng prevent rotavirus infection in newborn mice. $J$. Microbiol. Biotechnol. 28: 391-396.
30. Joo EJ, Chun J, Ha YW, Ko HJ, Xu MY, Kim YS. 2015. Novel roles of ginsenoside $\mathrm{Rg} 3$ in apoptosis through downregulation of epidermal growth factor receptor. Chem. Biol. Interact. 233: 25-34.

31. Kang LJ, Choi YJ, Lee SG. 2013. Stimulation of TRAF6/ TAK1 degradation and inhibition of JNK/AP-1 signaling by ginsenoside $\mathrm{Rg} 3$ attenuates hepatitis $\mathrm{B}$ virus replication. Int. J. Biochem. Cell Biol. 45: 2612-2621.

32. Kang S, Song MJ, Min H. 2018. Antiviral activity of ginsenoside $\operatorname{Rg} 3$ isomers against gammaherpesvirus through inhibition of p38- and JNK-associated pathways. J. Functional Foods 40: 219-228.

33. Cho A, Roh YS, Uyangaa E, Park S, Kim JW, Lim KH, et al. 2013. Protective effects of red ginseng extract against vaginal herpes simplex virus infection. J. Ginseng Res. 37: 210-218.

34. O'Donnell CD, Shukla D. 2008. The importance of heparin sulfate in herpesvirus infection. Virol. Sin. 23: 383-393.

35. Uchida H, Chan J, Goins WF, Grandi P, Kumagai I, Cohen JB, et al. 2010. A double mutation in glycoprotein gB compensates for ineffective $\mathrm{gD}$-dependent initiation of herpes simplex virus type 1 infection. J. Virol. 84: 12200-12209. 